\title{
Calculating Ionization Transition Rate for Circularly Polarized Fields, Including Non-Zero Initial Momentum
}

\author{
V.M. Ristić*, T.B. Miladinović and M.M. Radulović \\ Department of Physics, Faculty of Science, Kragujevac University, Kragujevac, Serbia
}

\begin{abstract}
Potassium atoms in circularly polarized laser field whose intensity $(I)$ varies from $2 \times 10^{12} \mathrm{~W} / \mathrm{cm}^{2}$ to $2.5 \times 10^{14} \mathrm{~W} / \mathrm{cm}^{2}$ were studied. In the case when there is zero initial momentum, transition rate (that depends only on $I$ ) exhibits standard behaviour: as $I$ increases, so thus the rate, until it reaches its maximum value at $1.1 \times 10^{14} \mathrm{~W} / \mathrm{cm}^{2}$; after that, rate diminishes as $I$ increases. In the case of non-zero initial momentum, transition rate (that now depends on $I$ but additionally on initial momentum, too) exhibits following behaviour: dependence of the rate on $I$ follows standard pattern, it rises with increase of $I$ until it reaches its maximum, and then diminishes. But with increase of momentum, ionization rate gradually diminishes.
\end{abstract}

PACS numbers: 32.80.-t, 32.80.Fb, 32.80.Rm

\section{Introduction}

In last thirty years there have been numerous papers that studied the interaction between an atom and strong laser electromagnetic fields. Opposite to multiphoton ionization which is dominant for high frequency laser fields, one of often used models for low frequency laser fields was based on the tunneling regime: external field leads to the formation of a barrier through which electron can tunnel out. The connection between two regimes can be determined by the Keldysh parameter [1]. In atomic units $\left(e=m_{\mathrm{e}}=\hbar=1\right)$, it has the following form $\gamma=\omega / \omega_{\mathrm{t}}=\omega \sqrt{2 E_{\mathrm{i}}} / F$, where $\omega$ is frequency of laser field, $\omega_{t}$ is tunneling frequency, $F$ is strength of laser field, $E_{\mathrm{i}}$ ionization energy. For $\gamma>1$, multiphoton regime prevails, for $\gamma \ll 1$, tunneling ionization occurs.

Thus electron tunneling through a potential barrier is one of the most important ionization mechanisms in strong low frequency external fields and has been extensively investigated - theoretical framework first was developed by Keldysh, and was later extended by Perelomov, Popov and Terent'ev. They obtained a formula for ionization rate of a bound state because of its interaction with external electromagnetic field (generated by laser) in 1966 [2]. In 1986, Ammosov, Delone and Krainov formulated ADK theory [3], extending the results of preceding theory to complex atoms and ions by calculating exactly the pre-exponent factor. Their formula for ionization rate by linearly polarized field is [3]:

\footnotetext{
* corresponding author; e-mail: ristic@kg.ac.rs
}

$$
W_{\mathrm{ADK}}^{\operatorname{lin}}=\left(\frac{4 Z^{3} e}{F n^{*^{4}}}\right)^{2 n^{*}-1} \exp \left(-\frac{2 Z^{3}}{3 F n^{*^{3}}}\right),
$$

where $Z$ is charge state, $n^{*}=Z / \sqrt{2 E_{\mathrm{i}}}$ is effective principal quantum number.

Formula given above was used as basis for calculations performed in numerous papers that dealt with different aspects of tunneling ionization $([4,5]$, and references therein). In actual calculations it was usually assumed that initial momentum of ejected electron was zero.

In recent years, there have been papers that dealt with non-zero momentum of ejected electrons [6-8]. Formula for transition rate in this case (for linearly polarized laser field) has the following form [4]:

$$
\begin{aligned}
& W_{\mathrm{pADK}}^{\operatorname{lin}}=\left(\frac{4 Z^{3} e}{F n^{*^{4}}}\right)^{2 n^{*}-1} \\
& \quad \times \exp \left(-\frac{2 Z^{3}}{3 F n^{*^{3}}}-\frac{p^{2} \gamma^{3}}{3 \omega}\right) .
\end{aligned}
$$

In this paper we are mainly concerned with behavior of the transition rate for circularly polarized field. Ionization rate in this case differs from rates for linearly polarized light by the factor $\sqrt{3 F Z^{3} / \pi n^{*^{3}}}$.

\section{Calculating non-zero initial momentum}

Transition rate formula for circularly polarized field (in cases of zero momentum) was obtained earlier [5, 9]

$$
\begin{aligned}
& W_{\mathrm{ADK}}^{\mathrm{cir}}=\sqrt{\frac{3 F Z^{3}}{\pi n^{*^{3}}}}\left(\frac{4 Z^{3} e}{F n^{*^{4}}}\right)^{2 n^{*}-1} \\
& \times \exp \left(-\frac{2 Z^{3}}{3 F n^{*^{3}}}\right) .
\end{aligned}
$$

Finally, including the non-zero initial momentum in 
above formula leads to the improved expression for transition rate in tunneling ionization by circularly polarized field

$$
\begin{gathered}
W_{\mathrm{pADK}}^{\mathrm{cir}}=\sqrt{\frac{3 F Z^{3}}{\pi n^{*^{3}}}}\left(\frac{4 Z^{3} e}{F n^{*^{4}}}\right)^{2 n^{*}-1} \\
\quad \times \exp \left(-\frac{2 Z^{3}}{3 F n^{*^{3}}}-\frac{p^{2} \gamma^{3}}{3 \omega}\right) .
\end{gathered}
$$

In order to perform necessary calculations, we needed an expression for momentum, which we obtained in paper [8] led by results of paper [10, 11]. There, the expression for momentum was given by

$$
p(\eta)=\sqrt{-\frac{1}{4}+\frac{1}{2 \eta}+\frac{1}{4 \eta^{2}}+\frac{F}{4} \eta} .
$$

Developing it further into power series leads to following:

$$
\begin{aligned}
& p(\eta)=\frac{1}{2}\left(\sqrt{F \eta-1}+\frac{1}{\eta \sqrt{F \eta-1}}+\ldots\right) \\
& \text { outside barrier } \eta>\frac{1}{F} .
\end{aligned}
$$

For a field intensity of $2 \times 10^{12} \mathrm{~W} / \mathrm{cm}^{2}$ lower limit for barrier has following value (atomic unit system): $\eta_{\mathrm{L}}=1 / F\left[2 \times 10^{12}\right]=189.802[7,8]$.

\section{Estimating the transition probability for circularly polarized laser fields with non-zero momentum included}

We studied transition rates for ionization of potassium atoms in circularly polarized electromagnetic field, both without and with non-zero momentum, see Eqs. (3), (4). Field intensities have been varied from $10^{12} \mathrm{~W} / \mathrm{cm}^{2}$ to $10^{16} \mathrm{~W} / \mathrm{cm}^{2}$ (at higher intensities the relativistic effects start to occur). The shape of the curved surface in the case of zero initial momentum is in its well-known form (there the rate does not depend on $\eta$ ), see Fig. 1. But in the case of non-zero initial momentum the rate depends on $\eta$, namely its value decreases with increase of $\eta$; see Fig. 1, the surface that is under the standard surface.

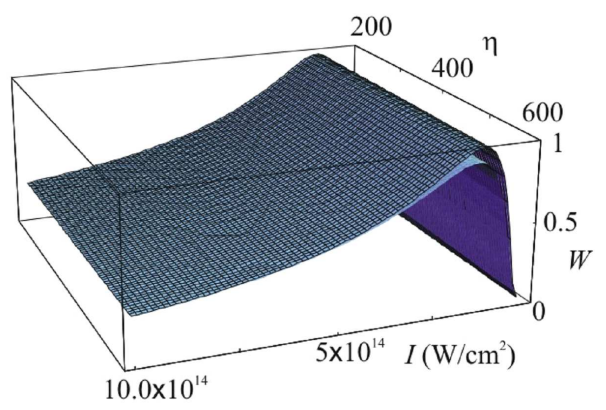

Fig. 1. Dependence of transition rates $W_{\mathrm{ADK}}^{\mathrm{cir}}$ and $W_{\mathrm{pADK}}^{\text {cir }}$ on field intensity, for zero initial momentum and non-zero initial momentum, respectively. The figure is rotated around $z$-axis in order to easier distinguish between given surfaces.
As for the transition rate maxima, it can be seen from Fig. 2 (that shows enlarged areas of interest from Fig. 1) that there is considerable decrease in the value in the case of non-zero initial momentum.
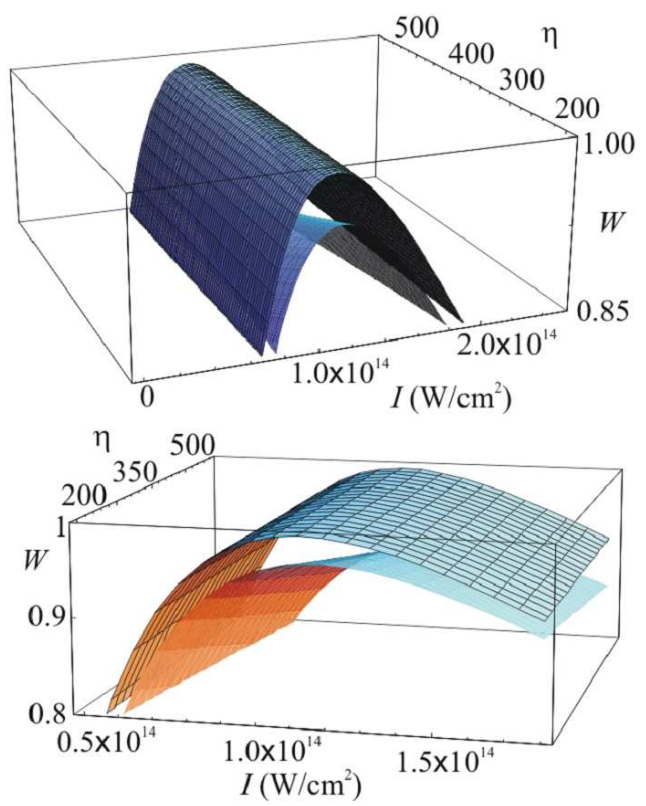

Fig. 2. Transition rates $W_{\mathrm{ADK}}^{\mathrm{cir}}$ and $W_{\mathrm{pADK}}^{\mathrm{cir}}$ dependence on field intensity, for zero initial momentum and non-zero initial momentum, respectively (enlarged part of Fig. 1).

In Fig. 3 there are given both maxima of transition rate, for fixed value of $\eta$ at 190. The Figure is a two-dimensional graph of data represented in three dimensions in the upper part of Fig. 2, and it shows more clearly the influence of the initial momentum on maximum of probability.

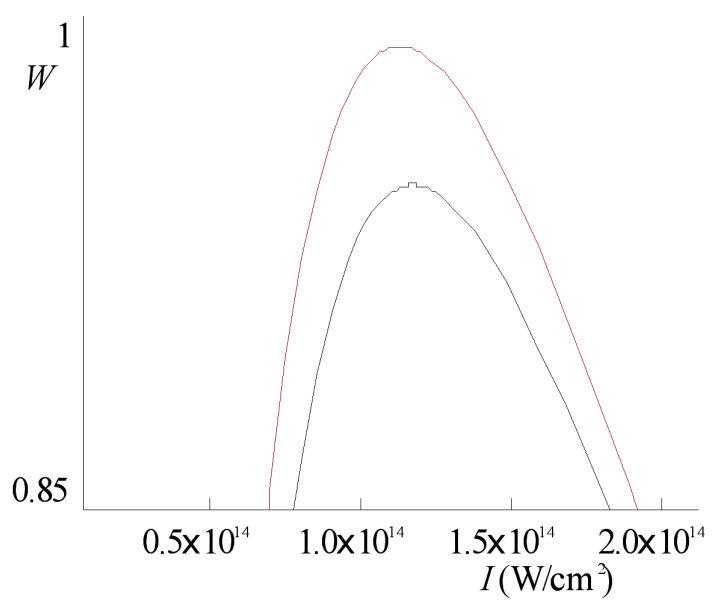

Fig. 3. Two-dimensional graphic of transition rates $W_{\mathrm{ADK}}^{\text {cir }}$ and $W_{\mathrm{pADK}}^{\text {cir }}$, for zero (upper curve) and non-zero (lower curve) momentum, for $\eta$ fixed at 190. 


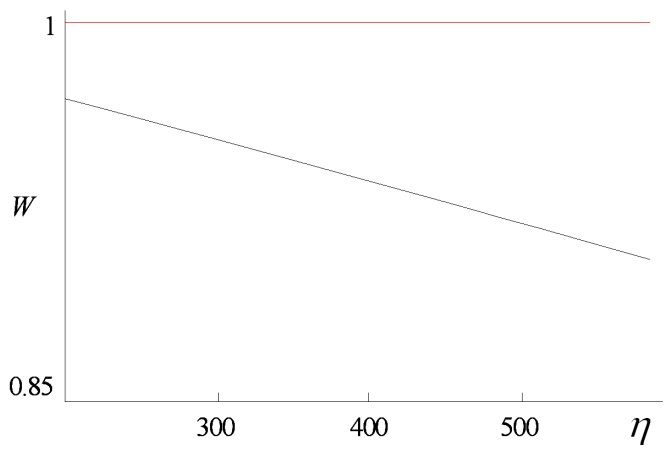

Fig. 4. Two-dimensional graphic of transition rates $W_{\mathrm{ADK}}^{\text {cir }}$ and $W_{\mathrm{pADK}}^{\text {cir }}$, for zero (upper curve) and non-zero (lower curve) momentum, for $I$ fixed at $1.1 \times$ $10^{14} \mathrm{~W} / \mathrm{cm}^{2}$.

In Fig. 4 there also can be seen both maxima in $2 \mathrm{D}$ graphic, only now we fixed field intensity at $1.1 \times$ $10^{14} \mathrm{~W} / \mathrm{cm}^{2}$. Just as in the case of linearly polarized field [8], transition rate decreases as value of momentum increases, see Fig. 4.

\section{Final remarks}

It was shown that besides standard behaviour circularly polarized laser field has anomalous behavior when non-zero initial momenta are included into transition rate calculation. It is manifested in Fig. 2, where the surface presenting transition rate has indicative tent shape, showing that transition rate decreases as $\eta$ increases. As $\eta$ is representing the momentum of the ejected electron, it can be said that our earlier conclusions are confirmed [7], i.e. that part of laser pulse energy is used for increasing momenta of ejected electrons, leaving smaller amounts of light quanta available for ionization of remaining electrons.

As electron tunneling through a potential barrier is one of the most important ionization mechanisms in strong low frequency external fields the result we obtained could be very interesting for further research.

\section{Acknowledgments}

This work was supported in part by the Ministry of Science, Republic of Serbia (Project 141023).

\section{References}

[1] L.V. Keldysh, Sov. Phys. JETP 20, 1307 (1965).

[2] A.M. Perelomov, V.S. Popov, M.V. Terent'ev, Sov. Phys. JETP 23, 924 (1966).

[3] V.M. Ammosov, N.B. Delone, V.P. Krainov, Sov. Phys. JETP 64, 1191 (1986).

[4] V.P. Krainov, V.M. Ristić, Sov. Phys. JETP 101 , 1479 (1992).

[5] N.B. Delone, V.P. Krainov, Phys. Usp. 41, 469 (1998).

[6] V.M. Ristić, T.S. Premović, Kragujevac J. Sci. 25, 41 (2003).

[7] V.M. Ristić, T.B. Miladinović, M.M. Radulović, Acta Phys. Pol. A 112, 909 (2007).

[8] V.M. Ristić, T.B. Miladinović, M.M. Radulović, Laser Phys. 18, 10, 1 (2008).

[9] E. Gubbini, PhD Thesis, 2004.

[10] D. Bauer, Phys. Rev. A 55, 55 (1997).

[11] D. Bauer, Theory of Laser-Matter Interaction, Max-Planck Institute, Heidelberg 2002. 